\title{
Alterações morfofisiológicas em folhas de cafeeiro (Coffea arabica L.) consorciado com seringueira (Hevea brasiliensis Muell. Arg.)
}

\author{
Morphophysiological alternations in leaves of Cofeea arabica L. plants in consort with \\ Hevea brasiliensis Muell. Arg.
}

\author{
Erivaldo Alves do Nascimento ${ }^{1}$ Luiz Edson Mota de Oliveira ${ }^{2}$ Evaristo Mauro de Castro ${ }^{2}$ \\ Nelson Delú Filho ${ }^{3}$ Alessandro Carlos Mesquita ${ }^{4}$ Carlos Vinicio Vieira $^{5}$
}

\section{RESUMO}

O cultivo da seringueira em monocultivo ou consorciada com cafeeiro surge como alternativa promissora e uma opção para os cafeeicultores frente às constantes oscilações da produção e do mercado. Porém, a produtividade de ambas as culturas é fortemente afetada pelas variações climáticas e pelo sistema de cultivo adotado. $O$ objetivo deste trabalho foi avaliar os efeitos da variação dos fatores do clima e dos sistemas de cultivo sobre as trocas gasosas, eficiência fotoquímica do fotossistema II (FV/FM) e anatomia foliar do cafeeiro. Foram estudados quatro sistemas de cultivo: café em monocultivo (C), três fileiras de cafeeiros a cada fileira dupla de seringueira (SSCCCSS), uma fileira de cafeeiros a cada fileira de seringueira (SCS) e três plantas de café a cada planta de seringueira na mesma fileira (SCSCS). As plantas dos sistemas (SSCCCSS), (SCS) e (SCSCS) apresentaram os menores valores de taxas fotossintéticas (A), condutância estomática (gs), transpiração (E) e maiores valores para a razão $\mathrm{FV} / \mathrm{Fm}$. As plantas de café em (C) apresentaram médias superiores de espessura dos parênquimas paliçádico e lacunoso, do limbo foliar, além de maior índice estomático em relação aos demais sistemas de cultivo, apresentando, dessa forma, plasticidade anatômica para a espécie, quando comparada às plantas de sol e sombreadas pela seringueira.

Palavras-chave: sombreamento; anatomia; consórcio; fotossíntese.

\section{ABSTRACT}

One alternative to reduce the effects of the production instability and of the insecurity of world budget over coffee growers is the cultivation of rubber tree intercropped with coffee. However, the productivity of both cultures is affected strongly by the climatic variations and for the crop system. The objective of this research was to perform evaluations of gas exchanges, chlorophyll fluorescence (Fv/Fm ratio) and leaf anatomy of coffee trees in cropped in different systems with rubber tree and environment. Four intercropping systems were studied as described: full sun coffee (C), three coffee cultivation lines between double strip of rubber tree (SSCCCSS), one coffee cultivation line between rubber tree strips (SCS) and three coffee plants for one rubber tree in the same cultivation line (SCSCS). The coffee plants of the (SSCCCSS), (SCS) and (SCSCS) systems showed smaller values of net photosynthesis rate (A), stomatal conductance (gs) and transpiration (E) and highest values of $\mathrm{Fv} / \mathrm{Fm}$ ratio than that observed for the $(C)$ system. The coffee plants cropped in the $(C)$ system presented the highest average leaf thickness and also the largest thickness of palisade and lacunar parenchymas and stomatal index. These results indicate that coffee leaves have a great anatomic plasticity when cropped at full sun light or under shaded caused by rubber trees.

Key words: shading, anatomy, intercropping systems, photosynthesis.

\section{INTRODUÇÃO}

Entre as espécies de café, a de maior expressão econômica é a Coffea arabica L., por produzir o café mais apreciado em todos os países consumidores. A espécie é uma rubiácea que apresenta numerosas variedades e cultivares, oriunda da Etiópia, onde é cultivada em locais predominantemente

${ }^{1}$ Fisiologia Vegetal, Recife, PE, Brasil.

${ }^{2}$ Departamento de Biologia, Universidade Federal de Lavras (UFLA), Lavras, MG, Brasil.

${ }^{3}$ Universidade Vale do Rio Verde, Três Corações, MG, Brasil.

${ }^{4}$ Departamento de Biologia da UFLA, Setor de Fisiologia Vegetal, 37200-000, Lavras, MG, Brasil, Caixa Postal 3037. E-mail: mesquita@ufla.br. Autor para correspondência.

${ }^{5}$ Fisiologia Vegetal, UFLA, Lavras, MG, Brasil. 
sombreados, nas regiões montanhosas ocidentais, entre 1.000 e $2.500 \mathrm{~m}$ de altitude (MIRANDA et al., 1999). Embora seja considerada uma cultura umbrófila, o cafeeiro pode ser perfeitamente cultivado em pleno sol, uma vez que é capaz de desenvolver características fisiológicas e anatômicas que permitem sua fotoaclimatação sob altos níveis de irradiância (RAMALHO et al., 1997).

O sistema de cultivo de cafeeiros consorciados com seringueiras surge como alternativa promissora e uma opção para os cafeicultores frente às constantes oscilações do café no mercado. A exploração de ambas as culturas, por meio de um sistema de cultivo adequado, intercalando-as, contribuirá para uma maior proteção do cafeeiro contra ventos frios, geadas e altas temperaturas, e além disso, possibilitará a geração de novos empregos diretos e fixos no campo e agregar uma fonte de renda extra para os cafeicultores.

O nível de radiação ao qual as plantas estão expostas pode afetar as características morfológicas, anatômicas e fisiológicas da folha, interferindo diretamente sobre o desenvolvimento, função e estrutura foliar, estrutura dos cloroplastos e componentes do processo fotossintético. As plantas, quando cultivadas em pleno sol, apresentam folhas bastante espessas devido ao desenvolvimento dos parênquimas paliçádico e esponjoso, o qual é induzido pela alta intensidade de luz, acarretando um aumento da área do mesofilo (BOARDMAN, 1977; CUI et al., 1991; ABRAMS et al., 1994; LEE et al., 2000).

A espessura das lâminas foliares e a densidade estomática podem variar de acordo com a localização da folha na planta e com o grau de exposição ao sol. Sendo a área foliar de plantas exposta à plena radiação, apresentará um menor limbo foliar quando comparada com as folhas de plantas sombreadas (PATTON \& JONES, 1989; ABRAMS et al., 1994; $\mathrm{KLICH}, 2000$ ).

No caso do cafeeiro, as características anatômicas são altamente influenciadas pelo nível de radiação. VOLTAN et al. (1992) constataram que o número de estômatos por $\mathrm{mm}^{2}$ e as espessuras dos parênquimas paliçádico e esponjoso decresceram com o aumento no nível de irradiância nos cultivares de $\boldsymbol{C}$. arábica Mundo Novo, Catuaí-Vermelho e Bourbon Vermelho, no cultivar de C. canphora Apoatã e no híbrido interespecífico Icatu Amarelo.

O objetivo do presente trabalho foi estudar as variações das características morfofisiológicas da folha do cafeeiro cultivar Mundo Novo em monocultivo e em diferentes sistemas de consórcio com seringueira.

\section{MATERIAL E MÉTODOS}

Este trabalho foi realizado em plantas de café (Coffea arabica L. cultivar Mundo Novo) plantadas há 16 anos na fazenda experimental do Ministério da Agricultura, localizada no município de Varginha-MG, a $864 \mathrm{~m}$ de altitude, latitude $21^{\circ} 33^{\prime} 10^{\prime \prime} \mathrm{S}$ e longitude $46^{\circ}$ 26' 20"W GRW. O clima da região caracteriza-se por apresentar duas estações bem definidas: seca, de abril a setembro, e chuvosa, de outubro a março.

$\mathrm{O}$ experimento foi constituído de quatro tratamentos: monocultivo de café $(\mathrm{C})$, uma fileira de cafeeiros a cada fileira de seringueira (SCS), três fileiras de cafeeiros a cada fileira dupla de seringueira (SSCCCSS) e o quarto tratamento foi constituído pelos cafeeiros conduzidos entre plantas de seringueira na mesma fileira, no sistema (SCS), onde três plantas de café foram plantadas a cada planta de seringueira (SCSCS); desta forma, os cafeeiros dos sistemas (SCS) e (SCSCS) foram consorciados com a mesma seringueira. $\mathrm{O}$ espaçamento utilizado para o cafeeiro em monocultivo foi de $3 \times 1 \mathrm{~m}$, totalizando uma densidade de 3.333 plantas por hectare. No sistema (SSCCCSS), os espaçamentos utilizados foram $3 \times 1 \mathrm{~m}$ para o cafeeiro e 12 × $3 \mathrm{~m}$ para a seringueira, com uma densidade total de 3.555 plantas por hectare. Nos sistemas (SCS) e (SCSCS), o espaçamento foi 6 x $1 \mathrm{~m}$ e 6 $\mathrm{x} 3 \mathrm{~m}$ para as plantas de café e seringueira, respectivamente. Em ambos os sistemas, a densidade total de plantio foi de 2.221 plantas por hectare.

Com o auxílio do Infrared gas analyzer (IRGA), foram avaliadas as seguintes características: transpiração, temperatura foliar, condutância estomática, fotossíntese, eficiência de carboxilação e fotoquímica (relação Fv/Fm). Essas avaliações foram realizadas em folhas completamente expandidas do terço superior da planta de café.

As características anatômicas avaliadas foram as espessuras das epidermes nas faces adaxial e abaxial; parênquimas esponjoso e paliçádico; número de células epidérmicas $\mathrm{mm}^{-2}$; número de estômatos $\mathrm{mm}^{-}$ ${ }^{2}$ e diâmetros polar e equatorial dos mesmos.

As características anatômicas da seção transversal do limbo foliar foram obtidas de um total de cinco folhas completamente expandidas do terço superior das plantas de café, utilizando-se três campos por cada folha, totalizando um total de 15 repetições. Posteriormente, estas folhas foram fixadas em FAA-70 (formaldeído + ácido acético + álcool etílico 70\%) por 72 horas, seguindo a metodologia de JOHANSEN (1940) e, posteriormente, conservadas em álcool etílico $70^{\circ} \mathrm{GL}$.

Os cortes transversais foram efetuados à mão livre, com o auxílio de lâminas de barbear e inclusão 
do material em pecíolo de imbaúba, sendo estes submetidos à clarificação em solução de hipoclorito de sódio a $20 \%$ de produto comercial, por um período de três a cinco minutos, seguido de três lavagens em água destilada. Posteriormente, foram submetidos ao processo de coloração com azul de astra-safranina, seguindo-se os métodos descritos por KRAUS \& ARDUIM(1997). Os cortes foram montados em lâminas semipermanentes com água glicerinada.

Com relação à caracterização dos estômatos, foram feitos cortes paradérmicos manuais, utilizandose lâminas de barbear. Os cortes foram realizados na parte mediana de cada folha, na epiderme da face abaxial, e submetidos ao processo de coloração diretamente na lâmina com safranina a $0,1 \%$ em água glicerinada. As observações foram realizadas com o auxílio de câmara clara, em microscópio Olympus CBB, segundo a técnica de LABORIAU et al. (1961). Em cada região da lâmina foliar, foram observados quatro campos, totalizando 40 campos por tratamentos (10 folhas por tratamento).

O delineamento experimental utilizado foi inteiramente casualizado. Os dados obtidos foram submetidos às análises de variâncias, sendo que as médias foram comparadas pelo teste de Tukey, a 5\% de probabilidade de erro, utilizando-se o programa estatístico SISVAR versão 4.3 (FERREIRA, 2000).

\section{RESULTADOS E DISCUSSÃO}

As taxas de transpiração, temperatura, condutância estomática, fotossíntese líquida, eficiência fotoquímica $(\mathrm{Fv} / \mathrm{Fm})$ determinadas nos cafeeiros da espécie C.arábica estão demonstradas na tabela 1 . Nota-se que o aumento no sombreamento provoca significativas reduções na taxa fotossintética, na temperatura foliar, na condutância estomática e na relação $\mathrm{Fv} / \mathrm{Fm}$. Segundo revisão realizada por DA MATTA (2004), as baixas temperaturas afetam a fotossíntese do cafeeiro e a sua produção através dos fatores estomáticos e não estomáticos. Além disso, a concentração interna de $\mathrm{CO}_{2}$ em plantas de café aumenta logariticamente até $30^{\circ} \mathrm{C}$ e mais rapidamente até $35^{\circ} \mathrm{C}$, indicando desta forma uma limitação térmica na fotossíntese.

Plantas de C.arábica cv. Catuai Vermelho apresentam valores baixos de transpiração, condutância estomática e fotossíntese líquida em plantas cultivadas isoladamente $\left(1.39 \mu \mathrm{mol} \mathrm{m} \mathrm{m}^{-2} \mathrm{~s}^{-1}, 0.09 \mu \mathrm{mol} \mathrm{m} \mathrm{m}^{-2} \mathrm{~s}^{-1} \mathrm{e}\right.$ $2.32 \mu \mathrm{mol} \mathrm{m} \mathrm{m}^{-2} \mathrm{~s}^{-1}$, respectivamente), segundo CARVALHO et al. (2001). Entretanto, o mesmo autor cita que esses valores são relativamente baixos se comparados aos descritos por BIERHUIZEN et al. (1969), YAMAGUCHI \& FRIEND (1979), KUMAR \& TIESZEN (1980) e CARELLI et al. (1999).

Taxas de fotossíntese máxima em folhas de café em pleno sol, em torno de $7 \mu \mathrm{mol} \mathrm{CO} \mathrm{m}^{-2} \mathrm{~s}^{-1}$, foram relatadas por CANNELL (1985), sendo este valor semelhante ao obtido por KUMAR E TIEZEN (1980), quando obtiveram uma fotossíntese máxima em torno de $1,4 \mathrm{~g} \mathrm{CO} \mathrm{m}^{-2} \mathrm{~h}^{-1}$, (equivale a $8,8 \mu \mathrm{mol} \mathrm{m}^{-2} \mathrm{~s}^{-1}$ ). CARELLI et al. (1999) não encontraram diferenças entre fotossíntese e condutância estomática em plantas de C. arábica e C. robusta crescidas em condições de pleno sol e sombreadas artificialmente (50\%), contudo, a fotossíntese e a condutância estomática decresceram nas espécies com $80 \%$ de sombreamento. Esses resultados corroboram os demonstrados na tabela 1 , principalmente na redução da fotossíntese em plantas de café nos consórcios (SCS) e (SCSCS).

Os valores de Fv/Fm demonstram que as plantas de café mantidas em monocultivo apresentaram

Tabela 1 - Valores médios para transpiração $(\mathrm{T})$, temperatura foliar $\left(\mathrm{T}^{\circ} \mathrm{C}\right)$, condutância estomática (gs), fotossíntese (A) e eficiência fotoquímica $(\mathrm{Fv} / \mathrm{Fm})$.

\begin{tabular}{lccccc}
\hline Sistemas de cultivo & $\mathrm{E}\left(\mathrm{mmol} \mathrm{m}^{-2} \mathrm{~s}^{-1}\right)$ & $\mathrm{T}^{\circ} \mathrm{C}$ & $\mathrm{Gs}\left(\mathrm{mmol} \mathrm{m}^{-2} \mathrm{~s}^{-1}\right)$ & $\mathrm{A}\left(\mu \mathrm{mol} \mathrm{m}^{-2} \mathrm{~s}^{-1}\right)$ & $\mathrm{Fv} / \mathrm{Fm}$ \\
\hline C & $1,405 \mathrm{a}$ & $33,81 \mathrm{a}$ & $0,45 \mathrm{a}$ & $4,08 \mathrm{a}$ & $0,0243 \mathrm{a}$ \\
SSCCCSS & $0,8125 \mathrm{ab}$ & $30,02 \mathrm{~b}$ & $0,425 \mathrm{a}$ & $1,43 \mathrm{ab}$ & $0,049 \mathrm{~b}$ \\
SCS & $0,495 \mathrm{~b}$ & $29,14 \mathrm{bc}$ & $0,25 \mathrm{a}$ & $0,382 \mathrm{~b}$ & $0,013 \mathrm{~b}$ \\
SCSCS & $0,317 \mathrm{~b}$ & $28,4 \mathrm{c}$ & $0,125 \mathrm{~b}$ & $0,352 \mathrm{~b}$ & $0,011 \mathrm{~b}$ \\
\hline
\end{tabular}

As médias seguidas da mesma letra não diferem pelo teste de Tukey a 5\% de probabilidade de erro. (C) representa monocultivo; (SSCCCSS), três fileiras de cafeeiros a cada fileira dupla de seringueira; (SCS) uma fileira de cafeeiros a cada fileira de seringueira e (SCSCS), três plantas de café plantadas a cada planta de seringueira. 
maior eficiência do sistema antena para a absorção e transferência de energia luminosa, o que é comprovado pelos valores de fotossíntese líquida (Tabela 1).

O cafeeiro cultivado em pleno sol, no sistema (C), apresentou as maiores médias em espessura do limbo foliar e dos parênquimas paliçádico e esponjoso (Tabela 2). Por outro lado, observou-se que não houve diferenças entre os sistemas de cultivos com relação às epidermes da superfície abaxial e adaxial. Este aumento do parênquima paliçádico e, conseqüentemente, do limbo foliar constitui característica importante ao processo fotossintético. Segundo BOLHAR-NORDENKAMPF \& DRAXLER (1993), folhas com parênquima paliçádico mais espesso apresentam coeficiente de extinção da luz mais alto, portanto, espera-se que tais folhas tenham maior taxa fotossintética, conforme observado no presente trabalho (Tabela 1). Resultados semelhantes foram obtidos em trabalhos realizados com diferentes cultivares de $\boldsymbol{C}$. arabica, inclusive com a cultivar Mundo Novo, por VOLTAN et al. (1992) e FAHL (1994), que verificaram que as plantas cultivadas a $50 \%$ e $30 \%$ da luz solar apresentaram reduções de $6,5 \%$ e $13 \%$, respectivamente, na espessura total do limbo foliar. Verificaram também que as plantas cultivadas sob o nível do $100 \%$ de luz solar apresentaram maior espessura dos parênquimas paliçádico e esponjoso.

O sistema de cultivo em pleno sol (C) apresentou médias superiores no número de estômatos por $\mathrm{mm}^{-2}$ e número de células por $\mathrm{mm}^{-2}$ (Tabela 3 ). Neste sistema de cultivo, verificou-se também o maior índice estomático. Com relação ao diâmetro polar, não se observaram diferenças entre os sistemas de cultivo. Contudo, o diâmetro equatorial não diferiu entre os sistemas de cultivo (C), (SSCCCSS) e (SCSCS), sendo que o sistema (SCS) foi superior aos demais.

Em seu trabalho de revisão, DA MATTA (2004) cita que condições severas podem ser associadas com a aclimatização ou adaptação para a alta irradiância em café, além de exemplos citados por outros autores, como o aumento da densidade estomática (VOLTAN et al., 1992); o decréscimo da área foliar específica e a espessura da epiderme com mais cera epicuticular (VOLTAN et al., 1992; FAHL et al., 1994).

O processo de aclimatação envolve um aumento no "quencking" não fotoquímico, que aparentemente constitui uma linha primária de defesa contra a alta irradiância (RAMALHO et al., 1997 e 2000). Fato este novamente observado com a relação Fv/Fm em plantas de café conduzidas em monocultivo (Tabela 1).

A quantidade, distribuição, tamanho, forma e mobilidade dos estômatos são características específicas de cada espécie e podem ser alteradas em função das adaptações às condições ambientais (LARCHER, 2000).

Nota-se uma correlação positiva entre a densidade estomática (Tabela 3) e a assimilação de $\mathrm{CO}_{2}$ (Tabela 1). Esses resultados corroboram as observações de ABRAMS et al. (1994), segundo as quais a densidade estomática geralmente está positivamente relacionada com as trocas gasosas, pois o aumento da densidade estomática está associado com maior condutância estomática (BOARDMAN, 1977).

Os valores mais altos do índice estomático encontrados no sistema de cultivo (C) podem ser atribuídos a uma diferença de demanda evaporativa do ar entre os diferentes sistemas de cultivo. $O$ índice

Tabela 2 - Espessura da epiderme da superfície abaxial, adaxial, parênquima paliçádico, parênquima esponjoso e limbo foliar no cafeeiro cultivar Mundo Novo em diferentes sistemas de cultivo (média de 20 repetições).

\begin{tabular}{|c|c|c|c|c|c|}
\hline Sistema de cultivo & $\begin{array}{c}\text { Epiderme } \\
\text { abaxial }(\mu \mathrm{m})\end{array}$ & $\begin{array}{c}\text { Epiderme } \\
\text { adaxial }(\mu \mathrm{m})\end{array}$ & $\begin{array}{l}\text { Esp. Par. Paliçádico } \\
(\mu \mathrm{m})\end{array}$ & $\begin{array}{l}\text { Esp. Par. lacunoso } \\
\qquad(\mu \mathrm{m})\end{array}$ & $\begin{array}{c}\text { Espessura do } \\
\operatorname{limbo}(\mu \mathrm{m})\end{array}$ \\
\hline $\mathrm{C}$ & $39,05 \mathrm{a}$ & $40,16 \mathrm{a}$ & $151,13 \mathrm{a}$ & $328,51 \mathrm{a}$ & $558,85 \mathrm{a}$ \\
\hline SSCCCSS & $31,10 \mathrm{a}$ & $48,27 \mathrm{a}$ & $79,30 \mathrm{~b}$ & $299,87 \mathrm{~b}$ & $458,54 \mathrm{~b}$ \\
\hline SCS & $33,06 \mathrm{a}$ & $67,11 \mathrm{a}$ & $68,27 \mathrm{~b}$ & $302,18 \mathrm{~b}$ & $470,62 \mathrm{~b}$ \\
\hline SCSCS & $28,13 \mathrm{a}$ & $44,14 \mathrm{a}$ & $76,24 \mathrm{~b}$ & $285,76 \mathrm{~b}$ & $434,27 \mathrm{~b}$ \\
\hline
\end{tabular}

As médias seguidas da mesma letra não diferem pelo teste de Tukey a 5\% de probabilidade de erro. (C) representa monocultivo; (SSCCCSS), três fileiras de cafeeiros a cada fileira dupla de seringueira; (SCS) uma fileira de cafeeiros a cada fileira de seringueira e (SCSCS), três plantas de café plantadas a cada planta de seringueira. 
Tabela 3 - Valores médios do número de estômatos, número de células, índice estomático, diâmetro polar e equatorial dos estômatos no cafeeiro cultivar Mundo Novo em diferentes sistemas de cultivo (média de 40 repetições).

\begin{tabular}{|c|c|c|c|c|c|}
\hline Sistemas de cultivo & $\begin{array}{l}\mathrm{N}^{\mathrm{o}} \text { de estômatos/ } \\
\mathrm{mm}^{-2}\end{array}$ & $\begin{array}{c}\mathrm{N}^{\mathrm{o}} \text { de células/ } \\
\mathrm{mm}^{-2}\end{array}$ & $\begin{array}{c}\text { Índice estomático } \\
(\%)\end{array}$ & $\begin{array}{l}\text { Diâmetro polar } \\
(\mu \mathrm{m})\end{array}$ & $\begin{array}{l}\text { Diâmetro equatorial } \\
\qquad(\mu \mathrm{m})\end{array}$ \\
\hline $\mathrm{C}$ & $197,21 \mathrm{a}$ & $941,28 \mathrm{a}$ & $17,42 \mathrm{a}$ & $27,09 \mathrm{a}$ & $17,30 \mathrm{~b}$ \\
\hline SSCCCSS & $132,46 \mathrm{~b}$ & $846,93 \mathrm{~b}$ & $13,60 \mathrm{~b}$ & $26,72 \mathrm{a}$ & $16,82 \mathrm{~b}$ \\
\hline SCS & $127,65 \mathrm{~b}$ & $741,11 \mathrm{~d}$ & $14,69 \mathrm{~b}$ & $27,16 \mathrm{a}$ & $18,69 \mathrm{a}$ \\
\hline SCSCS & $125,80 \mathrm{~b}$ & $795,87 \mathrm{c}$ & $13,48 \mathrm{~b}$ & $26,53 \mathrm{a}$ & $17,06 \mathrm{~b}$ \\
\hline
\end{tabular}

As médias seguidas da mesma letra não diferem pelo teste de Tukey a 5\% de probabilidade de erro. (C) representa monocultivo; (SSCCCSS), três fileiras de cafeeiros a cada fileira dupla de seringueira; (SCS) uma fileira de cafeeiros a cada fileira de seringueira e (SCSCS), três plantas de café plantadas a cada planta de seringueira.

estomático é razoavelmente constante para uma dada espécie, sendo afetado principalmente pela umidade (CUTTER, 1986). Em relação ao diâmetro polar, não foi encontrada, neste trabalho, uma relação entre essa variável anatômica e as trocas gasosas.

Nos cortes paradérmicos, observa-se a forte influência do sombreamento sobre as dimensões das células epidérmicas e sobre a densidade estomática nas folhas do cafeeiro, pois observa-se, nas tabelas $2 \mathrm{e}$ 3 , que estas características variaram de acordo com o grau de sombreamento promovido pela seringueira.

A anatomia foliar é altamente especializada para a absorção de luz, sendo que as propriedades do mesofilo, principalmente as do parênquima paliçádico, garantem a absorção uniforme da luz através da folha. Segundo TAIZ \& ZEIGER (2004), em geral, as folhas de sol são mais espessas e apresentam células paliçádicas mais longas do que as de sombra. Contudo, o aumento na espessura da folha, especialmente pela elongação ou adição de células paliçádicas, tem sido associado a uma redução na resistência do mesofilo ao dióxido do carbono (NOBEL, 1977), fato este que pode levar a uma taxa fotossintética foliar mais elevada a pleno, em comparação com as plantas crescidas à sombra (BJORKMAM, 1981). Similarmente, VOLTAN et al. (1992), trabalhando com diferentes níveis de sombreamento, verificaram que o número de estômatos por $\mathrm{mm}^{2}$ no cultivar Mundo Novo decresceu linearmente com o nível de luz ao qual as plantas foram submetidas. Estes autores obtiveram valores médios de 256,17, 195,00 e 157,86 estômatos por $\mathrm{mm}^{2}$ em 100, 50 e $30 \%$ de luz solar, respectivamente. No entanto, eles verificaram que os diferentes níveis de radiação não tiveram influência sobre o comprimento e a largura dos estômatos.

As variações na anatomia foliar observadas neste trabalho se assemelham às de outras espécies, quando submetidas a diferentes regimes de luz (ZANELA, 2001; ALMEIDA, 2001; KLICH, 2000;
ABRAMS \& MOSTOLLER, 1995). ZANELA (2001) observou que plantas de Hymenaea courbaril, Malucra tinctoria e Acacia mangium, quando cultivadas a pleno sol, apresentam folhas mais espessas devido a maiores espessuras do mesofilo e dos parênquimas paliçádico e lacunoso.

\section{CONCLUSÃO}

O cafeeiro apresenta, em sua estrutura interna das folhas de sol e de sombra, uma grande amplitude de adaptação fenotípica como resultado do sombreamento produzido pela seringueira.

\section{AGRADECIMENTOS}

Ao Conselho Nacional de Desenvolvimento Científico e Tecnológico (CNPq), pela concessão de bolsa ao pesquisador Mesquita.

\section{REFERÊNCIAS}

ABRAMS, M.D. et al. Relating wet and dry year ecophysiology to leaf structure in constrasting temperate tree species. Ecology, Washington, v.75, n.1, p.123-133, 1994.

ABRAMS, M.D.; MOSTOLLER, S.A. Gas exchange, leaf structure and nitrogen in constrastin successsional tree species growing in open and understory sites during a drought. Tree Physiology, Victoria, v.15, n.6, p.361-370, 1995.

ALMEIDA, L.P. Germinação, crescimento inicial e anatomia foliar de plantas jovens de Cryptocarya aschersaniana MEZ. sob diferentes níveis de radiação. 2001. 96f. Dissertação (Mestrado em fisiologia vegetal) Universidade Federal de Lavras.

BIERHUIZEN, J.F. et al. Studies on the productivity of coffee. II. Effect of soil moisture on photosynthesis and transpiration of Coffea arabica. Acta Botanica Neerlandica, Amsterdam, v.18, p.367-374, 1969. 
BJORKMAN, O. Responses to different quantum flux densities. In: LANGE, O. et al. (eds.). Physiological plant ecology I. Responses to the physical environment. New York: Spínger-Verlag, Encyclopedia of Plant Physiology, 1981. p. $57-107$.

BOLHAR-NORDENKAMPF, H.R.; DRAXLER, G. Functional leaf anatomy. In: HALL, D.O. et al. Photosynthesis and production in a changing environment. London: Chapenan e Hall, 1993. p.91-112.

BOARDMAN, N.K. Comparative photosynthesis of sun and shade plants. Annual Review of Plant Physiology, Palo Alto, v.28, p.355-377, 1977.

CANNELL, M.G.R. Physiology of coffee crop. In: CLIFFORD, M.N.; WILLSON, K.C. (Eds). C offee, botany, biochemistry and production of beans and beverage. London: Croom Helm, 1985. p.108-134

CARELLI, M.L.C. et al. Carbon isotope discrimination and gas exchange in Coffea species grown under different irradiance regimes. Revista Brasileira de Fisiologia Vegetal, Londrina, v.11, p.63-68, 1999.

CARVALHO, L.M. et al. Aspectos morfofisiológicos das cultivares de cafeeiro Catuaí-Vermelho e Conilon. Pesquisa Agropecuária Brasilieira, v.36, n.3, p.411-416, 2001.

CUI, M. et al. Chlorophyll and gradients in sun and shade leaves of Spinacia oleracea. Plant, Cell and Environment, Oxford, v.14, n.5, p.493-500, 1991

CUTTER, E.G. Anatomia vegetal. Traduzido por Gabriela Vera Maria Caruso Catena. Parte I. Células e tecidos. 2.ed. São Paulo: Rocca, 1986. 304p.

DA MATTA, F.M. Ecophysiological constrains on the production of shaded and unshaded coffee: a review. Field Crops Research, Amsterdam, v.86, n.2, p.99-114, 2004.

FAHL, J.I. et al. Nitrogen and irradiance levels affecting net photosynthesis and growth of Young coffe plants (Coffea arábica L.). Journal of Horticultural Science, Ahsford, v.69, n.1, p.161-169, 1994.

FERREIRA, D.F. Análise estatísticas por meio do Sisvar para Windows versão 4.0. In: REUNIÃO ANUAL DA REGIÃO BRASILEIRA DA SOCIEDADE INTERNACIONAL DE BIOMETRIA, 45., 2000, São Carlos, SP. Anais... São Carlos: Sociedade Internacional de Biometria, 2000. p.255-258.

JOHANSEN, D.A. Plant microtechnique. New York: Megraw-Hill, 1940. 523p.

KLICH, M.G. Leaf variations in Elaeagnus angustifolia related to environmental heterogeneity. Environmental and Experimental Botany, Elmsford, v.44, n.3, p.171-183, 2000

KRAUS, J.E.; ARDUIM, M. Manual básico de métodos em morfologia vegetal. Seropédica: Edur, 1997. 198p.
KUMAR, D.; TIESZEN, L.L. Photosynthesis in Coffea arabica. I. Effect of light and temperature. Experimental Agriculture Cambridge, v.16, p.13-19, 1980.

LABORIAU, L.G. et al. Transpiração de Schizolobium parahiba (Vell.). Toledo: Comportamento na estação chuvosa, nas condições de Caeté, Minas Gerais, Brasil. Anais da Academia Brasileira de Ciências, Rio de Janeiro, v.33, n.2, p.237257, 1961

LARCHER, W. Ecofisiologia vegetal. São Carlos: Rima, 2000. $531 \mathrm{p}$

LEE, D.W. et al. Effects of irradiance and spectral quality on leaf structure and function in seedlings of two southeast Asian Hopea (Dipterocarpaceae) species. American Journal of Botany, Columbus, v.87, n.4, p.447-455, 2000.

MIRANDA, E.M. de. et al. Comportamento de seis linhagens de café (Coffea arábica L.) em condições de sobreamento e a pleno sol no estado do Acre, Brasil. Ciência e Agrotecnologia, Lavras-MG, v.23, n.1, p.62-69, 1999.

NOBEL, P.S. Internal leaf area and cellular $\mathrm{CO}_{2}$ resistance: Photosynthetic implication of variations with grown conditions and plant species. Physiologia Plantarum, Copenhagem, v.40, n.2, p.137-144, 1977.

PATTON, L.; JONES, M.B. Some ralationships between leaf anatomy and photosynthetic characteristics of willows. New Phytologist, Cambridge, v.111, n.4, p.657-661, 1989.

RAMALHO, J.C. et al. Photosynthetic responses of Coffea arábica leaves to a short-term high light exposure in relation to $\mathrm{N}$ availability. Physilogia Plantarum, Copenhagen, v.101, n.1, p.229-239, 1997.

RAMALHO, J.C. et al. Photosynthetic acclimation to high light conditions in mature leaves of Coffeea arabica L.: role of xanthophylis, quenching mechanisms and nitrogen nutition. Aust. Journal Plant Physiology, v.27, p.43-51, 2000.

TAIZ, L.; ZEIGER, E. Fisiologia vegetal. Porto Alegre: Artmed, 2004. 719p.

VOLTAN, R.B.Q. et al. Variação na anatomia foliar de cafeeiros submetidos a diferentes intensidades luminosas. Revista Brasileira de Fisiologia Vegetal, São Carlos, v.4, n.2, p.99105, 1992

YAMAGUCHI, L.; FRIEND, D.J.C. Effect of leaf age and irradiance on photosynthesis of Coffea arabica Photosynthetica, v.13, p.271-278, 1979.

ZANELA, S.M. Respostas ecofisiológicas e anatômicas ao sombreamento em plantas jovens de diferentes grupos ecológicos. 2001. 79f. Dissertação (Mestrado em Fisiologia Vegetal) - Universidade Federal de Lavras. 